\title{
ENHANCEMENT OF STABILITY, RELEASE AND IN VITRO DIGESTIBILITY OF MULBERRY STEM
} EXTRACT USING MICROEMULSIONS

\author{
NATTAPORN SOONTHORNSIT ${ }^{1}$, WARINKARN HEMSTAPAT ${ }^{2}$, CHETSADAPORN PITAKSUTHEEPONG ${ }^{3}$, \\ TASANA PITAKSUTEEPONG ${ }^{*}$
}

${ }^{1}$ Department of Pharmaceutical Technology, Faculty of Pharmaceutical Sciences and Center of Excellence for Innovation in Chemistry, Naresuan University, Tha Pho, Mueang Phitsanulok, Phitsanulok 65000, Thailand, ${ }^{2}$ Department of Pharmacology, Faculty of Science, Mahidol University, Rama VI Road, Ratchathewi, Bangkok 10400, Thailand, ${ }^{3}$ Food Biotechnology Research Unit, National Center for Genetic Engineering and Biotechnology (BIOTEC), 113 Thailand Science Park, Phahonyothin Road, Khlong Nueng, Khlong Luang, Pathum Thani 12120, Thailand Email: tasanap@nu.ac.th

Received: 21 Mar 2017 Revised and Accepted: 19 May 2017

ABSTRACT

Objective: This study was aimed to develop oral microemulsions and to evaluate their ability to enhance stability, release and intestinal digestion of mulberry stem extract (MSE).

Methods: The pseudoternary phase diagrams were constructed using caprylic/capric triglyceride (oil), PEG-8 caprylic/capric glycerides (S), polyglyceryl-3 diisostearate $(\mathrm{CoS})$ and an aqueous phase. The effects of $\mathrm{S} / \mathrm{CoS}(\mathrm{Km})$ ratio and a cosolvent, i.e. polyethene glycol 400 or propylene glycol (PG), were investigated. The optimized formulations were selected and incorporated with MSE. Then, they were then subjected to stability, release and lipolysis studies. The control solution consisted of 50\% PG and 50\% water.

Results: The formation and characteristics of the microemulsions were influenced by Km and cosolvents. The two optimized formulations (F3 and F4) consisted of $10 \%$ oil, $70 \% \mathrm{~S} / \mathrm{CoS}$ mixture and $20 \%$ aqueous phase were chosen. The Km ratios of F3 and F4 were 4:1 and 3:1. The aqueous phase of F3 and F4 was water and water mixed with PG, respectively. These formulations could improve the stability of MSE better than the control solution. The accumulated release of MSE from F3, F4 and the control solution reached 100\% while that of unformulated crude extract reached only $70 \%$ after $6 \mathrm{~h}$. The lipolysis study showed that MSE incorporated in both F3 and F4 was digested more than double the percentage compared to that of MSE incorporated in the control solution.

Conclusion: MSE was successfully developed in microemulsions. They are shown to be promising vehicles for oral delivery of MSE. Further animal trials are suggested.

Keywords: Mulberry stem extract, Microemulsion, Stability, Release, In vitro intestinal digestion

(C) 2017 The Authors. Published by Innovare Academic Sciences Pvt Ltd. This is an open access article under the CC BY license (http://creativecommons.org/licenses/by/4.0/) DOI: http://dx.doi.org/10.22159/ijpps.2017v9i7.18609

\section{INTRODUCTION}

Various parts of the mulberry tree have long been widely used in Ayurvedic and various traditional medical systems [1]. The root bark is used as anti-tussive, anti-inflammatory and diuretic agents in Korea [2]. The leaves, fruit and bark have been used in traditional Chinese medicine to treat fever, improve eyesight, strengthen joints and lower blood pressure $[3,4]$. The leaves are also consumed in Korea, Japan and Chile as anti-hyperglycemic nutraceutical foods for patients with diabetes mellitus [5]. Recently, the ethanolic extract prepared from aerial parts of the mulberry tree has been shown to provide hypolipidemic, anti-inflammatory and antioxidative effects which helped in alleviating obesity-induced liver diseases and related metabolic disorder in rats [6]. However, the mulberry stems which are often pruned and cut as normal cultivation practice are rarely investigated for medicinal use.

Our previous study demonstrated the anti-inflammatory effects of MSE through the inhibition of nitric oxide, inducible nitric oxide synthase (iNOS) and cyclooxygenase (Cox)-2 productions in LPSactivated RAW 264.7 macrophages [7]. In addition, this extract has been investigated for antioxidant activities using various in vitro antioxidant assays [8]. It was shown to possess a hydrogen-donating ability, hydroxyl radicals quenching ability, superoxide scavenging activity, nitric oxide scavenging activity and iron reducing capacity [8]. It also has been investigated for the anti-nociceptive effect in anterior cruciate ligament transection (ACLT)-induced rat model of osteoarthritis (OA) [9]. Assessment of knee joint pain was performed by determining the change in the hind limb weight distribution between the operated and the non-operated contralateral limb using the hind limb weight bearing tester. The severity of cartilage damage to the knee joint on the operated limb was also observed using the modified Mankin grading system. It was found that oral administration of MSE at doses of 5.6, 56 and 560 $\mathrm{mg} / \mathrm{kg}$ for $8 \mathrm{w}$ significantly alleviated joint pain in a dose-dependent manner. MSE at $560 \mathrm{mg} / \mathrm{kg}$ also demonstrated to delay the progression of the articular cartilage degeneration. Considering these background studies, MSE has potential to be used as a dietary supplement for reducing OA pain or an alternative treatment for inflammatory and oxidative stress-related diseases.

The benefit of using plant crude extracts is that they contain complex mixtures of compounds which often contribute to the synergistic effect [10]. The stem of the mulberry tree has recently been reported to contain various polyphenolic constituents including flavonoids (morusin), stilbenoids (mulberroside, resveratrol and oxyresveratrol) and coumarins [2]. However, these polyphenols are also sensitive to heat and a lack of long-term stability $[11,12]$. From our previous study, the effect of temperature on the stability of pure compound oxyresveratrol and MSE was investigated [12]. It was observed that the degradation occurred as a function of temperature and time. The degradation of oxyresveratrol and the extract was more pronounced at high temperature $\left(45^{\circ} \mathrm{C}\right)$ and was greatly observed after $45 \mathrm{~d}$ of storage.

In addition, the solubility of mixtures of compounds is also a major challenge. The polyphenolic compounds are known to have poor oral bioavailability due to low aqueous solubility and low dissolution rate [13]. According to the biopharmaceutics classification system (BCS), most of polyphenols belong to class II (low solubility and high permeability) and class IV (low solubility and low permeability) [13]. 
Therefore, formulation of plant extracts in modern drug delivery systems in order to enhance the stability and dissolution/release rate of polyphenols in the gastrointestinal fluid was considered essential and thus was anticipated in this study. A formulation of particular interest is a microemulsion.

Microemulsions are isotropic dispersions of two immiscible liquids such as oil and water, stabilized by an interfacial film of surfactant and cosurfactant molecules [14]. These systems are thermodynamically stable, spontaneous formation, low viscosity and transparent appearance [14, 15]. As pharmaceutical drug delivery systems, microemulsions represent several advantages, including ease of preparation, thermodynamic stability, stabilizing of active ingredients and a long shelf-life [16-18]. It is also able to improve drug solubilization, keep the drug in solution form as it is travelling along gastrointestinal (GI) tract as well as enhance absorption in the intestinal tract [18]. The enhanced absorption effect of microemulsion in the intestinal tract is mainly due to the surfactant by disrupting the structure of the lipid bilayer and opening tight junction of intestinal epithelium $[18,19]$. Therefore, the aims of this study were to develop oral microemulsions and to evaluate their potential for enhancing the stability of incorporated MSE. In vitro release and intestinal lipolysis which were prognostic tools for oral absorption of the developed microemulsions containing MSE were also investigated.

\section{MATERIALS AND METHODS}

\section{Materials}

Oxyresveratrol, isolated and purified from Artocapus lakoocha heartwood or Puag-Haad, was prepared in-house using the method as previously described [12]. HPLC grade acetonitrile and methanol were purchased from RCI Labscan (Bangkok, Thailand). 4 bromophenylboronic acid, pancreatin from porcine pancreas, sodium taurodeoxycholate (NaTc), trizma maleate, L- $\alpha$-phosphatidylcholine and dimethylsulfoxide (DMSO) were purchased from Sigma-Aldrich (Saint Louis, Missouri, USA). Caprylic/capric triglyceride was purchased from Croda Singapore Pte., Ltd. (Singapore). PEG-8 caprylic/capric glycerides and polyglyceryl-3 diisostearate were from Gattefosse (Lyon, France). PG was purchased from Srichand United Dispensary Co., Ltd. (Bangkok, Thailand). Polyethylene glycol 400 (PEG 400) was purchased from Union Chemical 1986 Co. Ltd. (Bangkok, Thailand). Potassium dihydrogen phosphate, calcium chloride dihydrate $\left(\mathrm{CaCl}_{2} .2 \mathrm{H}_{2} \mathrm{O}\right)$ and sodium chloride were purchased from Merck (Darmstadt, Germany). Sodium hydroxide $(\mathrm{NaOH})$ was purchased from Ajax Finechem Pty. Ltd. (Seven Hills, Australia).

\section{Preparation of MSE}

Mulberry stems were supplied by the Queen Sirikit Sericulture Center, Tak Province, Thailand. Plant identification was done by Dr. Pranee Nangngam and the voucher specimen was deposited at the Faculty of Science, Naresuan University (Voucher Specimen No. 004067). The barks were removed, the stem woods were chopped into small pieces and air dried for $2 \mathrm{~d}$. The chopped stems continued drying at $45{ }^{\circ} \mathrm{C}$ for $2 \mathrm{~d}$ using a hot-air oven (Memmert, Schwabach, Germany). Then, they were extracted by the maceration technique using $80 \%$ ethanol at room temperature. The filtrate was filtered and evaporated under reduced pressure using a rotary evaporator (Buchi Rotavapor R-114, Flawil, Switzerland). The drying process was continued using a water bath (M25 Lauda, Deutschland, Germany). The percentage yield of the extract was calculated by the following equation:

$$
\text { Yield }(\%)=\left(\mathrm{Wt}_{2} / \mathrm{Wt}_{1}\right) \times 100
$$

Where $\mathrm{Wt}_{1}=$ weight in $\mathrm{g}$ of dried, chopped stem pieces and $\mathrm{Wt}_{2}=$ weight in $g$ of dried crude extract obtained.

\section{Determination of oxyresveratrol in MSE by HPLC analysis}

An isocratic reversed-phase HPLC method was performed to determine the amount of oxyresveratrol which was the bioactive compound and found in high amounts in MSE. An HPLC system (LC20AT, Shimadzu, Kyoto, Japan) consisted of a UV-Vis detector (SPD20A, Shimadzu, Kyoto, Japan), an auto-sampler (SIL-10ADVP, Shimadzu, Kyoto, Japan) and a column oven. A C18 boned-silica gel column (Gemini, $5 \mu \mathrm{m}, 150 \times 4.6 \mathrm{~mm}$, Phenomenex, Torrance, USA) was used. The mobile phase was acetonitrile mixed with $0.05 \mathrm{M}$ phosphate buffer pH 3 (1:3). The flow rate of $1 \mathrm{ml} / \mathrm{min}$ and column oven temperature of $30^{\circ} \mathrm{C}$ were set. The detection wavelength was $320 \mathrm{~nm}$.

MSE (10 mg) was dissolved in $10 \mathrm{ml}$ of $80 \%$ ethanol and then further diluted to $0.25 \mathrm{mg} / \mathrm{ml}$. The sample solution was mixed well, filtered through $0.45 \mu \mathrm{m}$ nylon membrane filters (Vertical Chromatography Co., Ltd., Bangkok, Thailand) and analyzed for oxyresveratrol by HPLC analysis. The amount of oxyresveratrol in the extract was calculated from the calibration curve equation.

\section{Construction of pseudoternary phase diagrams}

The pseudoternary phase diagrams of caprylic/capric triglyceride (oil), PEG-8 caprylic/capric glycerides (surfactant, S), polyglyceryl-3 diisostearate (cosurfactant, $\mathrm{CoS}$ ) and water were constructed to determine the area of microemulsions as previously described [20]. The weight ratio of $\mathrm{S} / \mathrm{CoS}$ (defined as $\mathrm{Km}$ ) varied as 2:1, 3:1 and 4:1. The effects of the cosolvents including PEG 400 and PG on the formation of microemulsions were also investigated. The cosolvent was added to the water phase at ratio 1:1. All components were weighed and mixed. Then they were allowed to equilibrate for at least $24 \mathrm{~h}$ before characterizing for microemulsions by visual inspection and polarized light microscopy. The formation of microemulsions was confirmed by observing the appearance (i.e. optically clear single-phase transparent) and birefringence under a microscope (CHS 1K 6281, Olympus Optical Co., Ltd., Tokyo, Japan) equipped with a cross-polarizer.

\section{Characterization of the selected unloaded microemulsions}

From the microemulsion regions in the pseudoternary phase diagrams, microemulsion formulations were chosen and their physical characteristics including particle size, viscosity and cloud point were determined. The average droplet size and polydispersity of microemulsion were detected by using ZetaPALS ${ }^{\circledR}$ (Brookhaven Instruments, New York, USA) at $25^{\circ} \mathrm{C}$. The viscosity of the formulations was measured by using a Brookfield DV-III programmable with CP40 cone and plate rheometer (Brookfield Engineering Laboratories Inc., Massachusetts, USA). Cloud point measurement was performed by visual observation in the temperature range 0 to $90{ }^{\circ} \mathrm{C}$. The temperature at which a microemulsion changes from transparent to turbid mixture was recorded as cloud point. All measurements were carried out in triplicate at $25^{\circ} \mathrm{C}$.

\section{Preparation of Microemulsions containing MSE}

The microemulsion formulations were incorporated with MSE at 2\% by weight and then subjected to further studies including stability test, release and intestinal lipolysis study. MSE dissolved in a solution consisting of $50 \%$ PG mixed with $50 \%$ water (the PG solution) was used as a control.

\section{Stability testing}

The chemical stability of MSE incorporated in the selected microemulsion formulations were investigated at $4{ }^{\circ} \mathrm{C}$, room temperature (RT) and $45^{\circ} \mathrm{C}, 75 \pm 5 \%$ relative humidity (RH) for 6 mo. The PG solution containing MSE was used as the control formulation. At each time point, the samples were accurately weighed and thoroughly mixed with an appropriate volume of $80 \%$ methanol. Then, they were centrifuged at 4,000 rpm for $10 \mathrm{~min}$. After that, the supernatant was further diluted with $80 \%$ ethanol and filtered through $0.45 \mu \mathrm{m}$ nylon membrane filter. The percentage remaining of MSE incorporated in all the formulations was detected based on the marker peak, i.e. oxyresveratrol, by HPLC analysis as detailed above. The HPLC analysis was performed in triplicate.

\section{In vitro release study}

The release of MSE from the tested formulations was determined using dissolution apparatus II. Briefly, $600 \mathrm{mg}$ of the selected microemulsion formulations or the PG solution containing MSE at $2 \%$ by weight were filled in hard gelatin capsules. The capsules were introduced into $900 \mathrm{ml}$ of a release medium (phosphate buffer solutions, $\mathrm{pH} 6.8$ ) at $37 \pm 0.5{ }^{\circ} \mathrm{C}$. The revolution speed of the 
dissolution apparatus paddle was kept constant at $50 \mathrm{rpm}$. Aliquots of $5 \mathrm{ml}$ were withdrawn from each vessel at selected time points and filtered through $0.45 \mu \mathrm{m}$ nitrocellulose membrane filters. An equal volume of fresh medium was replaced to maintain a constant total volume. The amount of MSE released was detected based on oxyresveratrol by HPLC analysis as detailed above. The test was performed in triplicate. The unformulated MSE powder at the equivalent amount presented in the tested formulations was also investigated for the in vitro release.

\section{In vitro intestinal lipolysis study}

Assessment of the digestibility of microemulsions is a preliminary indicator of in vivo bioavailability enhancement. The condition used for the in vitro lipolysis study was modified from Yin et al. [21]. The tested formulations containing MSE $(250 \mathrm{mg})$ were dispersed in $9 \mathrm{ml}$ of the digestive buffer $\left(50 \mathrm{mmol}\right.$ trizma ${ }^{\circledR}$ maleate, $150 \mathrm{mmol} \mathrm{NaCl}, 5$ $\mathrm{mmol} \mathrm{CaCl}{ }_{2} \cdot 2 \mathrm{H}_{2} \mathrm{O}$, pH 7.5) containing $5 \mathrm{mmol} \mathrm{NaTc}$ and $1.25 \mathrm{mmol} \mathrm{L-}$ $\alpha$-phosphatidylcholine in order to simulate the fasted intestinal conditions. The mixtures were incubated at $37{ }^{\circ} \mathrm{C}$ in a shaking water bath (model 1086, GFL Gesellschaft für Labortechnik mbH, Burgwedel, Germany). The digestion was then initiated by adding $1 \mathrm{ml}$ of pancreatin-containing the digestive buffer (final lipase concentration 1000 USP units $/ \mathrm{ml}$ ). The reaction was left under continuous stirring and $\mathrm{pH}$ was maintained at 7.5 by adding $0.5 \mathrm{M}$ $\mathrm{NaOH}$ solution. The progress of in vitro digestion was indirectly monitored by pH-stat. After $30 \mathrm{~min}$, the digestion was stopped by the addition of $90 \mu \mathrm{l} 0.5 \mathrm{M}$ 4-bromophenylboronic acid solution to inhibit lipase activity. The mixtures were centrifuged at 9,000 rpm for 20 min (Universal Refrigerated Centrifuge, Kubota Corporation, Tokyo, Japan) to separate the aqueous phase and the pellet phase. The aqueous phase was collected and then filtered through $0.45 \mu \mathrm{m}$ nitrocellulose membrane filters prior to HPLC analysis. The extent of the digestion was determined based on the amount of MSE detected in the aqueous phase compared to the initial amount added and was expressed as a percentage. Again, the amount MSE in the aqueous phase was detected based on oxyresveratrol by HPLC analysis as detailed above.

\section{Statistical analysis}

All data were expressed as mean \pm SD. Statistical analysis was determined using one-way analysis of variance (ANOVA) followed by the Tukey's test for multiple comparisons (GraphPad Prism 6.0, GraphPad Software Inc., San Diego, USA). P-values less than or equal 0.05 were considered significant.

\section{RESULTS AND DISCUSSION}

Various biological activities of MSE have been demonstrated in the previous studies [7-9]. However, long-term stability, water solubility and oral absorption of the crude extract were the major limitations to its application. Therefore, a drug delivery system is considered essential. Microemulsion formulation was selected to incorporate MSE in our study.

MSE was first prepared. It was obtained as a powder, which had a dark brown color, with a percentage yield of $5.13 \%$ of the raw woody material. The calculated amount of oxyresveratrol in MSE was $17.87 \pm 0.61 \%$.

\section{Pseudoternary phase diagram}

Caprylic/capric triglyceride was selected as the oil for our purposes as it is known to enhance the intestinal absorption of co-formulated drugs [22]. PEG-8 caprylic/capric glyceride is a well-defined mixture of 30\% mono-, di-and triglycerides of saturated C6-C14 fatty acids (predominantly C8 and C10 fatty acids), 50\% of mono-and di-fatty acid esters of polyethylene glycol and $20 \%$ of free PEG 400 [23], which was used as a surfactant given its effectiveness in enhancing the intestinal absorption of various drugs [24-26]. Polyglyceryl-3 di iso stearate was used as cosurfactant in this study.

The pseudoternary phase diagrams were constructed to investigate the optimum ratio of the four components including oil, surfactant, cosurfactant and aqueous phase. The constructed pseudoternary phase diagrams are presented in fig. 1 . Using the $\mathrm{Km}$ ratio of $1: 1$, the microemulsions could not form (data not shown). The microemulsions were obtained when the $\mathrm{Km}$ ratios were 2:1, 3:1 and $4: 1$ (fig. 1) and the area of the microemulsions observed was comparable. The effect of a cosolvent (i.e. PEG 400 and PG) on the formation of microemulsions was also investigated by fixing the $\mathrm{Km}$ ratio at $3: 1$. Either PEG 400 or PG was mixed with water at ratio 1:1 and used as the aqueous phase. The results showed that the addition of either PEG 400 or PG into the aqueous phase caused a reduction in the microemulsion area (fig. 2). The region of microemulsions with PEG 400 (fig. 2B) was less than that with PG (fig. 2C) and without the cosolvent (fig. 2A). It has been reported that the presence of hydrophilic molecules in the aqueous phase influences the optimal head group area of the surfactant by altering the solubility of the head group in the aqueous phase [26]. In this case, PEG 400 (MW 380-420 g/mole) has a higher molecular weight than PG (MW $76.09 \mathrm{~g} / \mathrm{mole}$ ), thus PEG 400 shows a higher negative effect on the formation of microemulsions than PG.

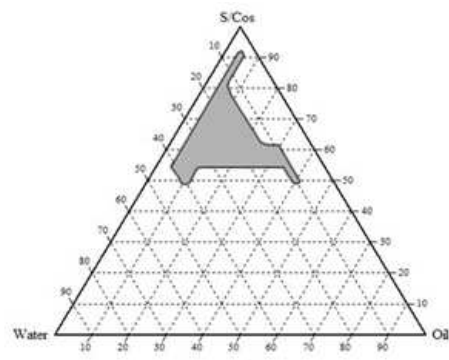

(A)

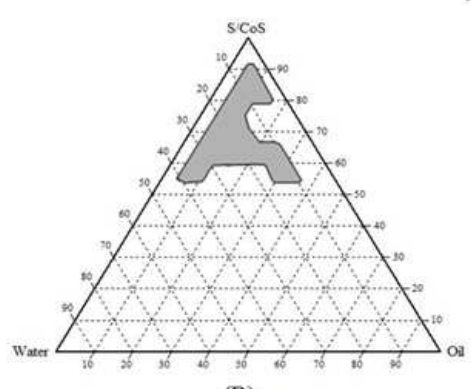

(B)

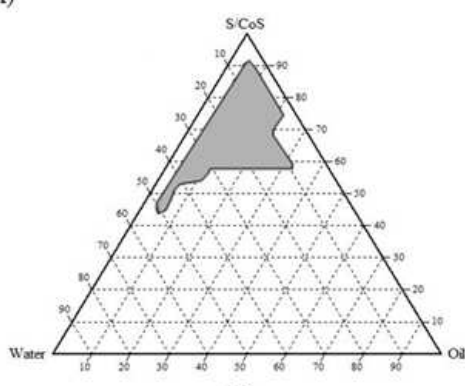

(C)

Fig. 1: Pseudoternary phase diagrams of caprylic/capric triglyceride (oil)/PEG-8 caprylic/capric (surfactant, S)/polyglyceryl-3 diisostearate (cosurfactant, CoS)/water with $\mathrm{Km}$ 2:1 (A), 3:1 (B) and 4:1 (C) 


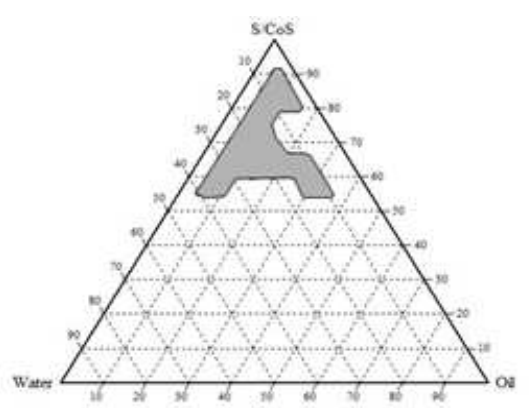

(A)

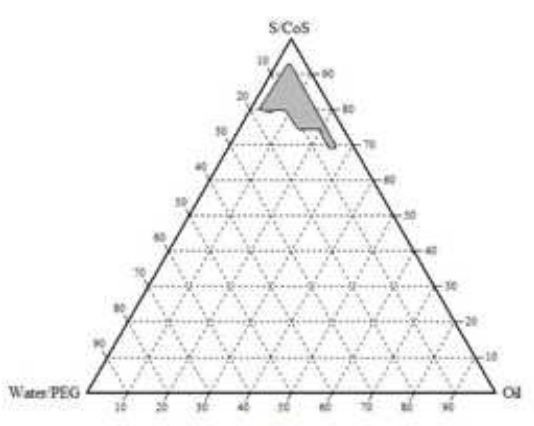

(B)

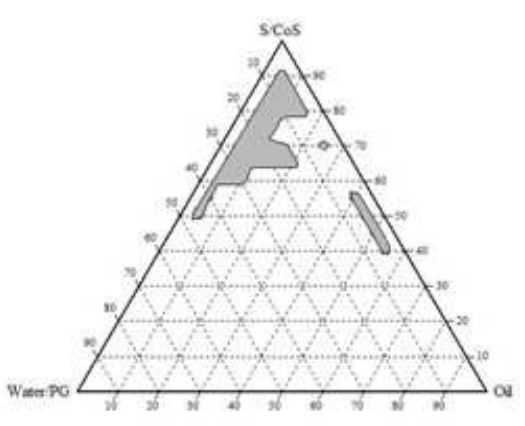

(C)

Fig. 2: Pseudoternary phase diagrams of caprylic/capric triglyceride (oil)/PEG-8 caprylic/capric glycerides: polyglyceryl-3 diisostearate $(\mathrm{Km}=3: 1) /$ aqueous phase. The aqueous phase was water $(\mathrm{A})$ or water mixed with PEG 400 (B) or PG (C) at the ratio of 1:1

The four unloaded formulations (F1-F4) were selected from the phase diagrams constructed. The compositions of each formulation are shown in table 1 . All formulations were characterised in terms of particle size, viscosity and cloud point. The results are shown in table 2. The mean droplets size range of microemulsion formulations was in the range of 3.27 to $7.60 \mathrm{~nm}$. The average viscosity of the formulations was 92-125 cP. The cloud point is an important property of non-ionic surfactants. It is the temperature at which the surfactants lose water solubility. The system becomes turbid or cloudy in appearance and phase-separates at this temperature. Thus, cloud point is important for determining the storage stability of microemulsions. The cloud point of unloaded microemulsion formulations followed the order: $\mathrm{F} 4>\mathrm{F} 3>\mathrm{F} 2>\mathrm{F} 1$. These results can be explained by the fact that, the formulations F3, F2 and F1 contained the same amount of components but different Km ratio, i.e. 4:1, 3:1 and $2: 1$, respectively. It is generally known that the higher hydrophilicity of a non-ionic surfactant (i.e. higher HLB), the higher the cloud point. In this study, PEG-8 caprylic/capric glycerides which were used as a surfactant has HLB $=14$ while polyglyceryl-3 di iso stearate which was used as a cosurfactant has HLB $=5$. Therefore, the higher Km ratio (i.e. 4:1 (HLB 12.2) >3:1 (HLB 11.8) >2:1 (HLB 11.0)) resulted in the higher cloud point. Comparing between $F 2$ and F4, it was observed that the presence of PG as a cosolvent in the aqueous phase of formulation $\mathrm{F} 4$ caused an increase in cloud point temperature compared to formulation F2. This can be explained by the fact that the solubility of the surfactant generally decreases with increasing temperature and starts to lose surface active properties. Turbid formulation is observed. However, the presence of the hydrophilic molecule, i.e. PG in the aqueous phase can increase the solubility of the head group of surfactant in the aqueous phase [27]. Thus, the solubility of surfactant can be remained and microemulsions can be stabilized.

Table 1: Percentage of components of the selected microemulsion formulations

\begin{tabular}{|c|c|c|c|c|}
\hline Components & F1 & F2 & F3 & F4 \\
\hline Caprylic/capric triglyceride & 10 & 10 & 10 & 10 \\
\hline PEG-8 caprylic/capric glycerides: polyglyceryl-3 diisostearate (2:1) & 70 & - & - & - \\
\hline PEG-8 caprylic/capric glycerides: polyglyceryl-3 diisostearate (3:1) & - & 70 & - & 70 \\
\hline PEG-8 caprylic/capric glycerides: polyglyceryl-3 diisostearate (4:1) & - & - & 70 & - \\
\hline Water & 20 & 20 & 20 & - \\
\hline Water: PG (1:1) & - & - & - & 20 \\
\hline
\end{tabular}

Table 2: Viscosity, particle size and cloud point of unloaded microemulsions

\begin{tabular}{llll}
\hline Formulations & Particle size $^{\mathbf{a}}(\mathbf{n m})$ & Viscosity $^{\mathbf{a}}$ (cP) & Cloud point $^{\left.{ }^{\circ} \mathbf{C}\right)}$ \\
\hline Unloaded F1 & $3.46 \pm 0.05$ & $125.53 \pm 0.69$ & 28 \\
Unloaded F2 & $3.40 \pm 0.08$ & $100.17 \pm 0.56$ & 37 \\
Unloaded F3 & $3.27 \pm 0.12$ & $92.10 \pm 0.70$ & 49 \\
Unloaded F4 & $7.60 \pm 0.12$ & $97.47 \pm 0.53$ & 60 \\
\hline
\end{tabular}

aValues are represented as mean \pm SD $(n=3)$ 


\section{Stability testing}

From the above results, $\mathrm{F} 1$ and $\mathrm{F} 2$ showed cloud point lower than $45^{\circ} \mathrm{C}$ and thus only F3 and F4 formulations were selected for further studies. The MSE was incorporated into these microemulsions at $2 \% \mathrm{w} / \mathrm{w}$ (namely MSE-F3 and MSE-F4). The PG solution containing MSE (namely MSE-PG solution) was used as the control. The results are shown in fig. 3. The degradation of MSE incorporated in F3 and $\mathrm{F} 4$, stored at $4{ }^{\circ} \mathrm{C}, 75 \%$ RH after 6 mo, was not significantly different and was shown to be the lowest (fig. 3A). However, significant degradation of MSE was observed in the PG solution compared with F4 microemulsion. The different in the ability to protect the degradation of MSE by various formulations was significantly observed when tested at a higher temperature. At RT, the degradation of MSE incorporated in both F3 and F4 was again not significantly different. However, both microemulsions have significantly enhanced the stability of MSE compared to the PG solution (fig. 3B). The percentage remaining of MSE loaded in both F3 and F4 stored at RT, $75 \%$ RH for 6 mo was higher than 86\%, suggesting that MSE-loaded microemulsions have good chemical stability. However, at $45^{\circ} \mathrm{C}$, the degradation of MSE increased. F4 showed the highest percentage remaining of MSE followed by F3 and the PG solution, respectively (fig. 3C). These results are in agreement with the cloud point temperature as previous discussed. F4 showed the highest cloud point, suggesting that it was the most thermodynamically stable microemulsion. Therefore, this may explain the most efficient performance of F4 to protect MSE degradation at high temperature.

\section{In vitro release}

The cumulative release of MSE from the selected microemulsion formulations and the PG solution (control) was detected again based on the content of oxyresveratrol using HPLC analysis. In the in vitro release study, the unformulated MSE powder was also investigated. From the release profiles during the first $60 \mathrm{~min}$ (fig. 4), it was observed that the profiles of the MSE-PG solution, MSE-F3 and MSEF4 showed immediate release because more than $75 \%$ of the active compound was released within 45 min [28]. The enhanced release rate was observed in MSE-F4, and was greater than the release rate of MSE-F3 and MSE-PG solution. The greater release rate of MSE incorporated in F4 may be attributed to the combination effect of the microemulsion and PG which is a well-known vehicle used to enhance dissolution of various drugs by cosolvent effect.
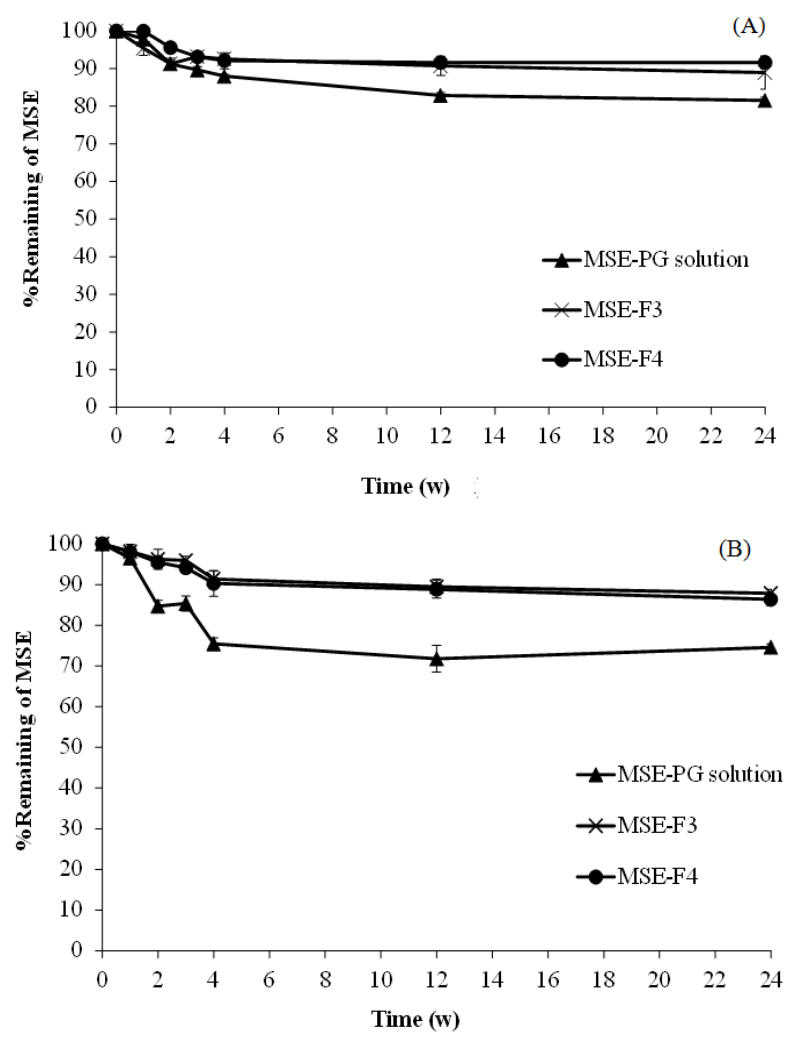

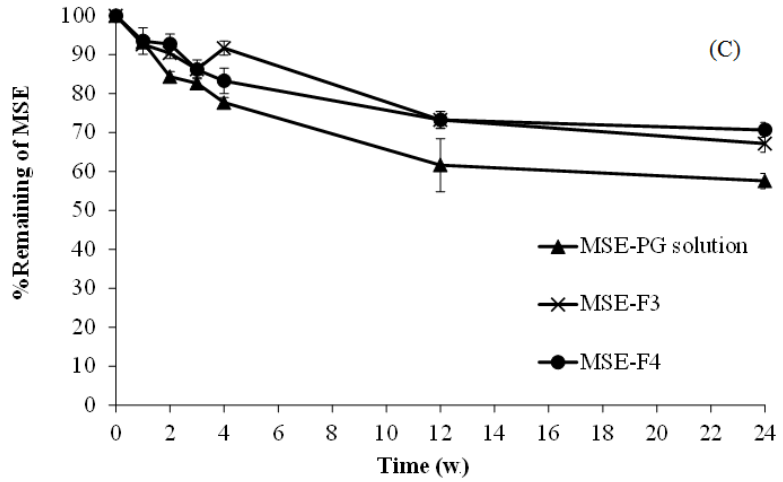

Fig. 3: The percentage remaining of mulberry stem extract formulated in propylene glycol solution (MSE-PG solution) and microemulsions (MSE-F3 and MSE-F4) after storing at (A) $4{ }^{\circ} \mathrm{C}$,

(B) RT and (C) $45^{\circ} \mathrm{C}$ for 6 mo. Values are represented as mean \pm SD $(n=3)$

The cumulative release of MSE from all three formulations reached $100 \%$ after $6 \mathrm{~h}$. However, the cumulative release of the unformulated MSE powder observed after $6 \mathrm{~h}$ was only $70 \%$. The superior release of MSE from the microemulsions compared to powder dosage form may be explained by the effect of surfactants which increase the solubility as well as the dissolution of MSE in the dissolution medium.

\section{In vitro intestinal lipolysis study}

In vitro intestinal lipolysis models, simulating the in vivo lipid digestion in the small intestine has been suggested as a promising tool to determine drug release from lipid-based formulations in a complex process taking place following ingestion of the formulations $[29,30]$. During lipolysis, MSE incorporated in a formulation may remain in the formulation, and be solubilized in the aqueous phase or precipitated out. The aqueous phase contains not only colloidal phases but also drug dissolves which is a prerequisite for absorption [30]. Thus, the percentage digestion of the formulations was determined based on the content of MSE in the aqueous phase. The results are shown in table 3. It was observed that digestibility of both microemulsion formulations was not significantly different. From the previous results, the in vitro release has been shown to release $100 \%$ of MSE from the microemulsion formulations and the PG solution after $6 \mathrm{~h}$. However, the percentage digestion of MSE incorporated in both microemulsion formulations increased by more than double the percentage compared to that in the PG solution (table 3). These results are in agreement with the findings of Gao et al. [31], who suggested that incorporation of cyclosporine A into the microemulsion could enhance its permeability through the intestinal membrane and oral bioavailability. The intestinal absorptionenhancing effect of the microemulsion may be explained by the fact that during lipolysis, lipids are digested by pancreatic enzymes resulting in monoglycerides, fatty acids and lysophospholipids etc [32]. These lipolytic products are arranged into micelles, unilamellar vesicles and multilamellar vesicles in the presence of bile salts and these structures enhance the solubilization and absorptive capacity of the small intestine. In addition, the enhanced intestinal absorption may be caused by surfactant-induced increment permeability which disrupts the structure of the lipid bilayer and opens the tight junction of the intestinal epithelium [18].

Table 3: Percentage digestion of the mulberry stem extract incorporated in the propylene glycol solution (MSE-PG solution) and the microemulsion formulations (MSE-F3 and MSE-F4)

\begin{tabular}{ll}
\hline Formulations & \% digestion \\
\hline MSE-PG solution & $31.85 \pm 0.99$ \\
MSE-F3 & $78.32 \pm 6.25^{*}$ \\
MSE-F4 & $83.03 \pm 6.21^{*}$ \\
\hline
\end{tabular}

Values are represented as mean $\pm S D(n=3)$. The designation * indicates significant difference compared with MSE-PG solution at $\mathrm{p}<0.05$ 


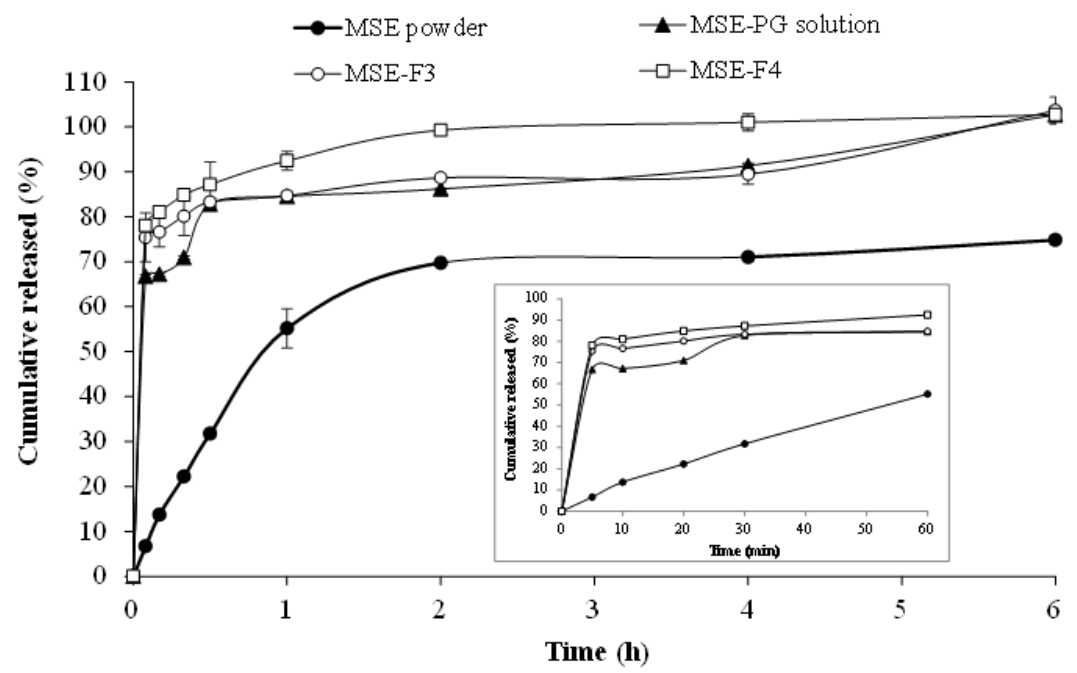

Fig. 4: In vitro release profile of the mulberry stem extract from various formulations including unformulated extract powder (MSE powder), propylene glycol solution (MSE-PG solution) and microemulsions (MSE-F3 and MSE-F4) in phosphate buffer solutions, pH 6.8. The insert showed the release profiles during the first $60 \mathrm{~min}$. Values are represented as mean $\pm S D(n=3)$

\section{CONCLUSION}

In this study, MSE was successfully developed in a modern delivery system, i.e. microemulsions. The two optimized formulations named F3 and F4 mainly consisted of $10 \% \mathrm{w} / \mathrm{w}$ caprylic/capric triglyceride, $70 \% \mathrm{w} / \mathrm{w}$ PEG-8 caprylic/capric glycerides and polyglyceryl-3 di iso stearate mixture and $20 \% \mathrm{w} / \mathrm{w}$ aqueous phase. The $\mathrm{Km}$ ratios of $\mathrm{F} 3$ and F4 were $4: 1$ and 3:1. The aqueous phase of F3 was water while that of $\mathrm{F} 4$ was water mixed with PG at the ratio of 1:1. MSE loading was $2 \% \mathrm{w} / \mathrm{w}$. MSE degradation was depending on the time of exposure, temperature and formulation. At RT and $45{ }^{\circ} \mathrm{C}$, MSE incorporated in both microemulsion formulations (i.e. MSE-F3 and MSE-F4) showed an improvement in the stability compared with that incorporated in the PG solution (control). The cumulative release of unformulated MSE powder was only $70 \%$ in $6 \mathrm{~h}$. However, the complete release of MSE was observed when formulated in these two microemulsion formulations. Although the complete release of MSE was achieved when formulated in the microemulsions and the PG solution, the percentage digestion of microemulsion formulations containing MSE was more than two times higher than the MSE-PG solution. These results suggest that the microemulsions containing MSE developed in this study are a promising vehicle for oral delivery of MSE. However, further studies in animal trials are required.

\section{ACKNOWLEDGEMENT}

The authors would like to acknowledge the financial support from the yearly budget of Naresuan University, Thailand (R2555B069 and R2558B053), the Center for Innovation in Chemistry (PERCH-CIC) and National Research Council of Thailand Graduate Student Scholarship (2014). We express special thanks to the Queen Sirikit Sericulture Center (Tak) for the supply of plant materials, and to Mr. Chein Yhirayha for the purification of the oxyresveratrol. Many thanks to Mr. Kevin Mark Roebl of the Naresuan University Language Centre for his editing assistance and advice on English expression in this manuscript.

\section{AUTHOR CONTRIBUTION}

Assoc. Prof. Tasana Pitaksuteepong is the Principal Investigator of the project and prepares the manuscript. Ms Nattaporn Soonthornsit is the graduate student who conducted the experiments. Asst Prof. Warinkarn Hemstapat helps to critically review and edits the manuscript. Dr. Chetsadaporn Pitaksutheepong helps in data analysis and critically reviews the manuscript.

\section{CONFLICT OF INTERESTS}

The authors declare that there is no conflict of interests regarding the publication of this article.

\section{REFERENCES}

1. Phukan P, Bawari M, Sengupta M. Promising neuroprotective plants from North-East India. Int J Pharm Pharm Sci 2015;7:28-39.

2. Chung KO, Kim BY, Lee MH, Kim YR, Chung HY, Park JH, et al. In vitro and in vivo anti-inflammatory effect of oxyresveratrol from Morus alba L. J Pharm Pharmacol 2003;55:1695-700.

3. Bown D. Encyclopedia of herbs and their uses. London: Dorling Kindersley; 1995.

4. Chan E WC, Lye PY, Wong SK. Phytochemistry, Pharmacology and clinical trials of Morus alba. Chin J Nat Med 2016;14:17-30.

5. Lemus I, Garcia R, Delvillar E, Knop G. Hypoglycaemic activity of four plants used in Chilean popular medicine. Phytother Res 1999;13:91-4.

6. Metwally FM, Ahmed HH, Rashad H, Zaazaa AM. Insights into the role of Morus alba in reversing obesity-associated steatosis and related metabolic disorder in rats. Asian J Pharm Clin Res 2016;9 Suppl 2:231-8.

7. Soonthornsit N, Pitaksutheepong C, Hemstapat W, Utaisincharoen P, Pitaksuteepong T. In vitro anti-inflammation activity of Morus alba L. stem extract in LPS-stimulated RAW 264.7 cells. Evid Based Complement Alternat Med 2017. Doi:10.1155/2017/3928956.

8. Pham PP, Morales NP, Pitaksuteepong T, Hemstapat W. Antioxidant activity of mulberry stem extract: a potential used as a supplement for oxidative stress-related diseases. Songklanakarin J. Sci. Technol; 2017. p. 7-31.

9. Khunakornvichaya A, Lekmeechai S, Pham PP, Himakoun W, Pitaksuteepong T, Morales NP, Hemstapat W. Morus alba L. stem extract attenuates pain and articular cartilage damage in the anterior cruciate ligament transection-induced rat model of osteoarthritis. Pharmacology 2016;98:209-16.

10. Kesarwani K, Gupta R. Bioavailability enhancers of herbal origin: an overview. Asian Pac J Trop Biomed 2013;3:253-66.

11. Munin A, Edwards-Levy F. Encapsulation of natural polyphenolic compounds; a review. Pharmaceutics 2011;3:793-829.

12. Yhirayha C. Formulation and skin penetration study of lyotropic liquid crystal incorporating mulberry stem (Morus alba L.) extract. Master thesis. M. Sc. Naresuan University, Phitsanulok; 2013.

13. Kaur H, Kaur G. A critical appraisal of solubility enhancement techniques of polyphenols. J Pharm 2014 http://dx.doi.org/10.1155/2014/180845

14. Eccleston GM. Microemulsions. In: Swarbrick J, Boylan J. editors. Encyclopedia of Pharmaceutical Technology. Marcel Dekker, New York; 1992. p. 375-421.

15. Kreilgaard M. Influence of microemulsions on cutaneous drug delivery. Adv Drug Delivery Rev 2002;54 Suppl 1:S77-S98. 
16. Liu DZ, Lecluyse EL, Thakker DR. Dodecylphosphocholinemediated enhancement of paracellular permeability and cytotoxicity in Caco-2 cell monolayers. J Pharm Sci 1999;88,1161-8.

17. Paul BK, Moulik SP. Uses and applications of microemulsions. Curr Sci 2001;80,990-1001.

18. Gundogdu E, Alvarez IG, Karasulu E. Improvement of the effect of water-in-oil microemulsion as an oral delivery system for fexofenadine: in vitro and in vivo studies. Int J Nanomed 2001;6:1631-40.

19. Zhong GG. Thermally Reversible microemulsion for oral delivery of poorly water-soluble drugs. In: Najjar R. editor. Microemulsions-An Introduction to properties and applications. Publisher: InTech; 2012. p. 135-50.

20. Noi-ang T, Charoensin A, Warangkanagool A, Soonthornsit N, Pitaksuteepong T. Development of oral microemulsions of Morus alba extract for osteoarthritis. Adv Mat Res 2015; 1060:41-4.

21. Yin YM, Cui FD, Mu CF, Choi MK, Kim JS, Chung SJ, et al. Docetaxel microemulsion for enhanced oral bioavailability: preparation and in vitro and in vivo evaluation. J Controlled Release 2009;140:86-94.

22. Swenson ES, Curatolo WJ. Intestinal permeability enhancement for proteins, peptides and other polar drugs: mechanisms and potential toxicity. Adv Drug Delivery Rev 1992;8:39-92.

23. Kreilgaard M, Pedersen EJ, Jaroszewski JW. NMR characterization and transdermal drug delivery potential of microemulsions. J Controlled Release 2000;69:421-33.

24. Rama Prasad YV, Puthli SP, Eaimtrakarn S, Ishida M, Yoshikawa $\mathrm{Y}$, Shibata N, et al. Enhanced intestinal absorption of vancomycin with labrasol and d- $\alpha$-tocopheryl PEG 1000 Succinate in rats. Int J Pharm 2003a; 250:181-90.

25. Rama Prasad YV, Eaimtrakarn S, Ishida M, Kusawake Y, Tawa R, Yoshikawa Y, et al. Evaluation of oral formulations of gentamicin containing labrasol in beagle dogs. Int J Pharm 2003b;268:13-21.

26. Koga K, Kusawake Y, Ito Y, Sugioka N, Shibata N, Takada K. Enhancing mechanism of labrasol on intestinal membrane permeability of the hydrophilic drug gentamicin sulfate. Eur J Pharm Biopharm 2006;64:82-91.

27. Lawrence MJ, Rees GD. Microemulsion-based on as novel drug delivery systems. Adv Drug Delivery Rev 2000;45:89-121.

28. Freire AC, Basit AW. Dissolution testing of solid dosage forms. In: Aulton ME, Taylor KMG. editors. Aulton's pharmaceutics: the design and manufacture of medicines. $4^{\text {th }}$ ed. London: Elsevier; 2013. p. 611-22.

29. Larsen AT, Sassene P, Mullertz A. In vitro lipolysis models as a tool for the characterization of oral lipid and surfactant-based drug delivery systems. Int J Pharm 2011;417:245-55.

30. Xiao L, Yi T, Liu Y, Zhou H. The in vitro lipolysis of lipid-based drug delivery systems: a newly identified relationship between drug release and lipid crystalline phase. Biomed Res Int 2016. http://dx.doi.org/10.1155/2016/2364317

31. Gao ZG, Choi HG, Shin HJ, Park KM, Lim SJ, Hwang KJ, et al. Physicochemical characterization and evaluation of a microemulsion system for oral delivery of cyclosporin A. Int J Pharm 1998;161:75-86.

32. Kalepu S, Manthina M, Padavala V. Oral lipid-based drug delivery systems an overview. Acta Pharm Sin B 2013;3:361-72.

\section{How to cite this article}

- Nattaporn Soonthornsit, Warinkarn Hemstapat, Chetsadaporn Pitaksutheepong, Tasana Pitaksuteepong. Enhancement of stability, release and in vitro digestibility of mulberry stem extract using microemulsions. Int J Pharm Pharm Sci 2017;9(7):103-109. 